\title{
Surface modification of stainless steel powders for microfabrication
}

\author{
M.T. Vieira ${ }^{a}$, A.G. Martins ${ }^{c}$, F.M. Barreiros ${ }^{a, b}$, M. Matos ${ }^{a}$, J.M. Castanho ${ }^{a, *}$ \\ a ICEMS-Materials and Surface Engineering Group, Mechanical Engineering Department, \\ Polo II, University of Coimbra, Coimbra, Portugal \\ ${ }^{\mathrm{b}}$ Centre for Rapid and Sustainable Product Development, Polytechnic Institute of Leiria, \\ Campus 5, Leiria, Portugal \\ c Instituto Pedro Nunes, Coimbra, Portugal
}

\section{A R T I C L E I N F O}

\section{Keywords:}

Coated powders

Stainless steel

Nanocrystallinity

Micromanufacturing

\begin{abstract}
A B S T R A C T
The current trend towards miniaturization has led to the increasing use of stainless steel powders as raw material in the manufacture small parts with complex shapes, e.g. medical devices. This paper focuses on the role of coated and uncoated low carbon stainless steel powders, having $d_{50}=7.3 \mu \mathrm{m}$, normal particle size distribution and a shape factor of 1 on additive processes used in micromanufacturing, e.g. micro powder injection moulding ( $\mu$ PIM). Surfaces were treated using a magnetron sputtering deposition apparatus equipped with a high frequency powder vibration and a continuous feed system. The results clearly show that the coated powders assume an "artichoke" morphology, which significantly increases the surface area. This combined with their nanocrystalline character leads to higher flowability than uncoated powders. However, no improvements have been observed concerning the critical powder volume concentration (CPVC) in feedstocks for $\mu$ PIM processing. In consolidation processes, the debinding temperatures can attain values higher than $500^{\circ} \mathrm{C}$. After debinding and sintering, the coating of steel powders can show lower carbon contamination in solid solution than uncoated ones. This result is very important particularly for powder microtechnology of low carbon stainless steel.
\end{abstract}

(c) 2007 Elsevier B.V. All rights reserved.

\section{Introduction}

The economical and technical advantages of powder metallurgy $(\mathrm{P} / \mathrm{M})$ processes have contributed to a significant increase in the use of sintered parts for functional and structural applications. In order to produce near-net-shape metal microcomponents of complex geometry in extended series, in a reliable and economic way, powder injection moulding (PIM) is one of the preferred techniques (German and Bose, 1997). This process applied to microforms ( $\mu$ PIM) is capable of pro- viding high precision and high performance properties after sintering (Liu et al., 2002, 2007; Zauner, 2006; Fu et al., 2004). $\mu$ PIM requires feedstocks composed of inorganic powders (raw material) and polymeric mixtures, in which the aim is to have the lowest quantity of polymeric binder capable of providing suitable viscosity in the mixing. Modification of the powder's surface could contribute to overcoming one or more problems inherent to this technique. Firstly, the technique should produce a more homogeneous mixture and decrease both the binder content in feedstocks (the ideal is to coat

\footnotetext{
* Corresponding author.

E-mail addresses: teresa.vieira@dem.uc.pt (M.T. Vieira), fbarreiros@estg.ipleiria.pt (A.G. Martins), agtmartins@ipn.uc.pt (F.M. Barreiros), mariana.matos@dem.uc.pt (M. Matos), jose.castanho@dem.uc.pt (J.M. Castanho). 0924-0136/\$ - see front matter @ 2007 Elsevier B.V. All rights reserved. doi:10.1016/j.jmatprotec.2007.11.162
} 
each particle with a thin layer of the binder system) and the debinding time after preconsolidation by injection moulding. Secondly, it should enhance sinter densities at low sintering temperatures, decreasing shrinkages and distortions. Thirdly, for stainless steel powders, it should lead to the reduction of carbon contamination in solid solution, thus decreasing sensitization phenomena. As well as particle size (S), particle size distribution (S) and the shape (S) of powders, it will be necessary to be aware to their surface (S) characteristics (structure and morphology). $3 \mathrm{~S}$ becomes $4 \mathrm{~S}$ as the dimensions of devices and parts decrease (Pujari, 1989; Hartwig et al., 1998; Scarlett, 2002). Better tailoring of surface powders can contribute to quality improvements in microparts and devices.

All surface powder modification processes that bring about surface changes to enable the production of sound components are welcome. Among powder surface modification processes, the sputtering technique could be one of the most promising, because it is highly efficient in producing powder coatings of different thicknesses, retaining or not their chemical composition, while at the same time decreasing significantly the crystallite size, which affects the interparticulate friction coefficient, wettability, reactivity and sintering mechanisms (Fernandes et al., 2003; Castanho et al., 2007).

This paper focuses on the characteristics of coated and uncoated low carbon stainless steel powders. The surface modification of powders was performed using magnetron sputtering equipment.

\section{Experimental details}

Gas-atomised (316L) stainless steel (SS) powders supplied by Osprey Metals (13.2\% Ni, 16.7\% Cr, 2.69\% Mo, 1.61\% Mn, 0.017\% C, $0.71 \% \mathrm{Si}$, balanced $\mathrm{Fe}$ ) were coated with similar chemical composition stainless steel, of different thicknesses, using a magnetron sputtering system with a modified substrate holder assisted by high frequency vibration. The deposition parameters were kept constant for all depositions as follows: target-to-substrate holder distance- $150 \mathrm{~mm}$; deposition pressure- $-0.5 \mathrm{~Pa}$; deposition power density $-16.7 \mathrm{~kW} / \mathrm{m}^{2}$ and unbiased. During the deposition process, a high frequency vibration supply ensured that the powders were continually in movement in the substrate holder so that a homogenous thin film would be deposited on each individual particle of stainless steel.

Particle size distribution was determined using a Coulter LS130 laser particle size analyzer. The morphology of the powders, before and after deposition and mixing with a polymer binder was observed using scanning electron microscopy (SEM). The as-deposited coating structures were also analysed by X-ray diffraction equipment (Philips X'Pert) using Co (K $\alpha)$ radiation, in a $\theta-2 \theta$ Bragg-Brentano mode and the grain size was calculated using a modified Scherrer equation. The flowability of powders was tested with a Flodex Hanson research flowmeter (Gioia, 1980). The thermal behaviour of the coated and uncoated powders in a reactive atmosphere was evaluated by TG-DTA SETARAM equipment.

As received (uncoated) and previously coated SS powders were mixed with an agar-based binder and a commercial binder, after optimising the powder/binder ratio. This optimi- sation was carried out with the uncoated and coated powders by measuring the mixing torque of different mixtures of stainless steel powders with agar-based or commercial binders, using a Bradender Plastograph, and the critical powder volume concentration (CPVC) evaluated and compared. The measuring principle of this technique is based on the resistance that material opposes to the rotation of the blades. In the methodology suggested in this study, after temperature stabilisation, the rotation speed of the measuring heads was selected and the system was switched on. The rotation speed adopted was $60 \mathrm{rpm}$, which was based on previous tests and on the observation that this value corresponded to the best commitment between the time required to stabilise the mixing torque and the effort needed to obtain an efficient mixture.

The binder was introduced into the mixing chamber in order to achieve a homogeneous temperature distribution. Then, a first fraction of the powder, corresponding to $50 \mathrm{vol} \%$, was added, followed by the successive addition of powder in order to attain a solid content in the mixture differing only $1 \%$ between several tests. In the feedstock based on the commercial binder, the mixture temperature was maintained constant at $150^{\circ} \mathrm{C}$. For the agar-based binder, the mixture temperature was $25^{\circ} \mathrm{C}$. A similar powder/binder ratio was selected for the coated and uncoated powders and both feedstocks were processed using the PIM methodology (preconsolidation, consolidation, debinding and sintering in conventional conditions) to distinguish the effect of the stainless steel powder's coating on the sensitization effect. In the latter case an optical microscope Nikon Optiphot was used to observe chemically attacked samples after polishing sinters resulting from coated and uncoated powders, using the same thermal cycle and surrounding atmosphere.

\section{Results and discussion}

A stainless steel thin film with similar chemical composition to the target was deposited on the powder's surface. The structure of powders was composed mainly of austenite and some martensite $\left(\alpha^{\prime}\right)$ and chromium carbides (Fig. 1(a)). However, the structure of thin films was completely ferritic as opposed to the target that is composed of a solid solution of austenite and primary chromium carbides. The X-ray diffraction pattern of the coated stainless steel powders exhibits diffraction of (110), (200) and (211) planes of a martensite- $\alpha^{\prime}$. This type of bcc structure visible on coatings deposited using austenitic stainless steel targets results from the sputtering technique, which induces a significant residual stress level in the coatings capable of transforming the austenite into a strain-induced martensitic phase transformations at temperatures $\left(M_{d}\right)$ higher than $25^{\circ} \mathrm{C}$ (Totten, 2007). Moreover, taking into consideration the broadening of the diffraction peaks, the coatings exhibit a nanocrystalline grain size (Fig. 1(b), detail).

The morphology of the uncoated and coated stainless steel (316L) powders is shown in Figs. 2(a and b), respectively. Due to the columnar growth the coating has an "artichoke/blunter" topography. The different types of densities are unchanged by the presence of a coating (Table 1). The influence of the coating's thickness is more effective in the thinnest coatings. In fact, the coated powders with lower deposition times studied 

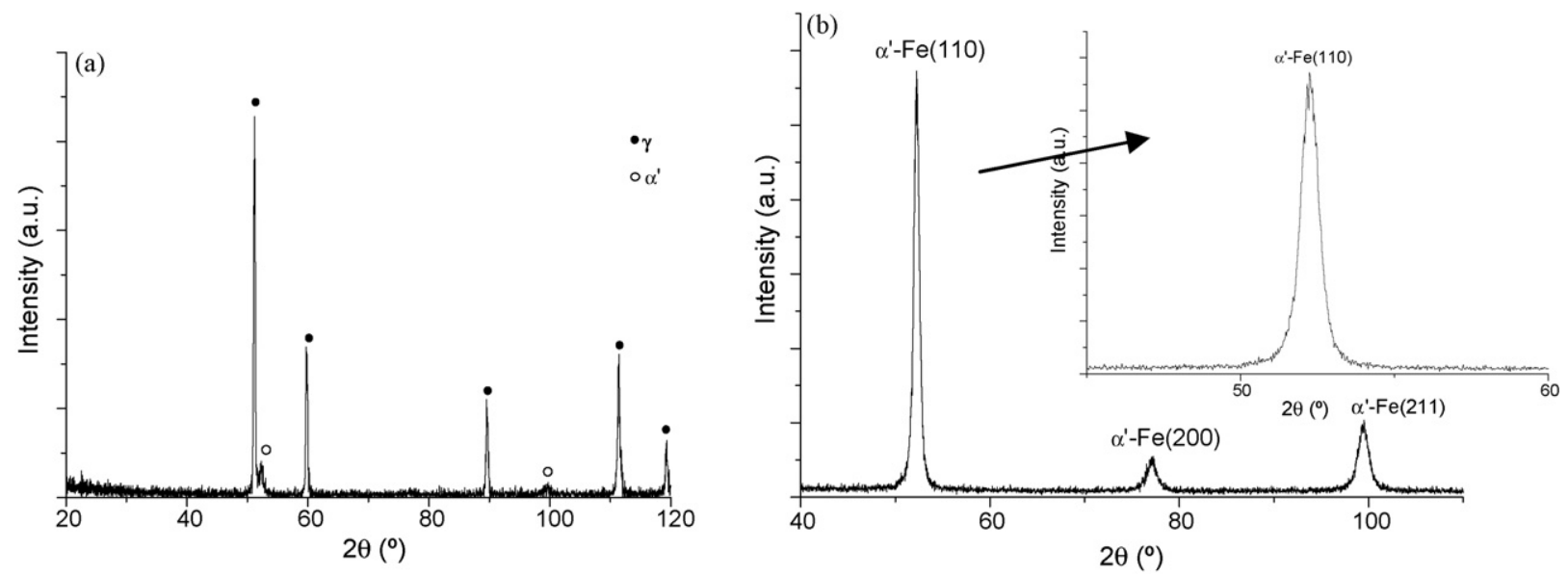

Fig. 1 - X-ray diffraction pattern of uncoated (a) and coated (b) stainless steel powders.
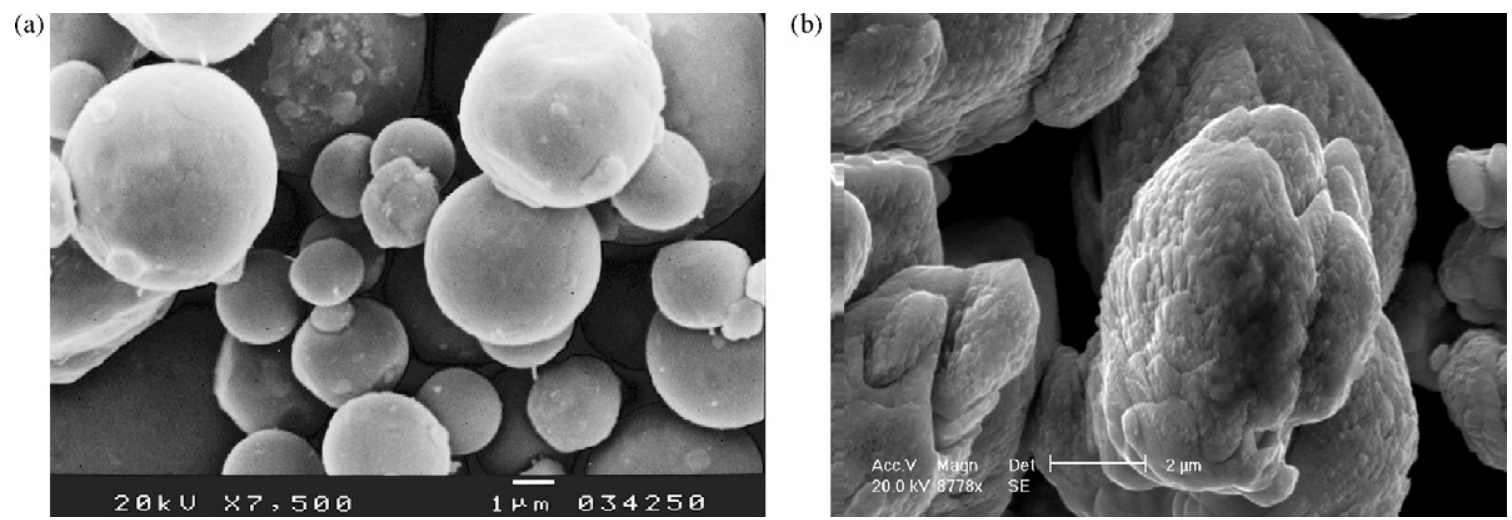

Fig. 2 - Morphology of the uncoated (a) and $8 \mathrm{~h}$ coated (b) stainless steel powders.

$-4 \mathrm{~h}-$ show higher tap density than the other coated powders, approaching that of uncoated ones, perhaps due to the particular morphology of the coating, which is more accentuated as thickness increases.

The modification of the powder surface induces a strong increase in flowability due to the decrease of interparticulate friction in the coated powders (Table 1). The highest flowability, measured by determining the smallest hole $-5 \mathrm{~mm}-$ that maintains the flow of the powders, was attained for the 4-h deposition. The interparticulate friction between the powders, which can be considered a qualitative measure of the cohesiveness of the powders, attains the lowest value for powders coated with the thinnest stainless steel thin films. Thus, if the only reason for coating powders were to improve their flow behaviour, a very thin film is enough to achieve such a purpose.

The thermal behaviour of the coated and uncoated powders in a reactive atmosphere, measured by DTA-TG, is presented in Fig. 3. TG analysis of the uncoated powders shows a gain in mass corresponding to a reaction process at $900^{\circ} \mathrm{C}$ and the DTA peak for this process is attained at $950^{\circ} \mathrm{C}$. The presence of a thin film increases the reactivity of the powder's surface decreasing the reaction temperature by about $150^{\circ} \mathrm{C}$. The mass gain in the coated powders, observed by TG graph occurs around $730^{\circ} \mathrm{C}$ and the DTA peak is attained at $800^{\circ} \mathrm{C}$.

The increase in reactivity of the coated powder's surface is due to the higher surface-to-volume ratio of the coated powders, which has various causes: the surface morphology is

\begin{tabular}{|c|c|c|c|c|}
\hline Powder & $\begin{array}{l}\text { Poured density } \\
\qquad\left(\mathrm{g} \mathrm{cm}^{-3}\right)\end{array}$ & $\begin{array}{l}\text { Tap density } \\
\left(\mathrm{g} \mathrm{cm}^{-3}\right)\end{array}$ & $\begin{array}{c}\text { Flowability } \\
\text { diameter }(\mathrm{mm})\end{array}$ & $\begin{array}{c}\text { Interparticulate } \\
\text { friction }(\mathrm{Pa})\end{array}$ \\
\hline Uncoated & $3.33 \pm 0.04$ & $4.38 \pm 0.01$ & 24 & $196 \pm 3$ \\
\hline $10 \mathrm{~h}$ coated & $3.30 \pm 0.02$ & $3.89 \pm 0.01$ & 6 & $48.6 \pm 0.3$ \\
\hline $8 \mathrm{~h}$ coated & $3.34 \pm 0.04$ & $4.12 \pm 0.01$ & 6 & $49.1 \pm 0.5$ \\
\hline $6 \mathrm{~h}$ coated & $3.42 \pm 0.03$ & $4.12 \pm 0.02$ & 7 & $58.7 \pm 0.5$ \\
\hline $4 \mathrm{~h}$ coated & $3.30 \pm 0.02$ & $4.23 \pm 0.01$ & 5 & $40.4 \pm 0.3$ \\
\hline
\end{tabular}




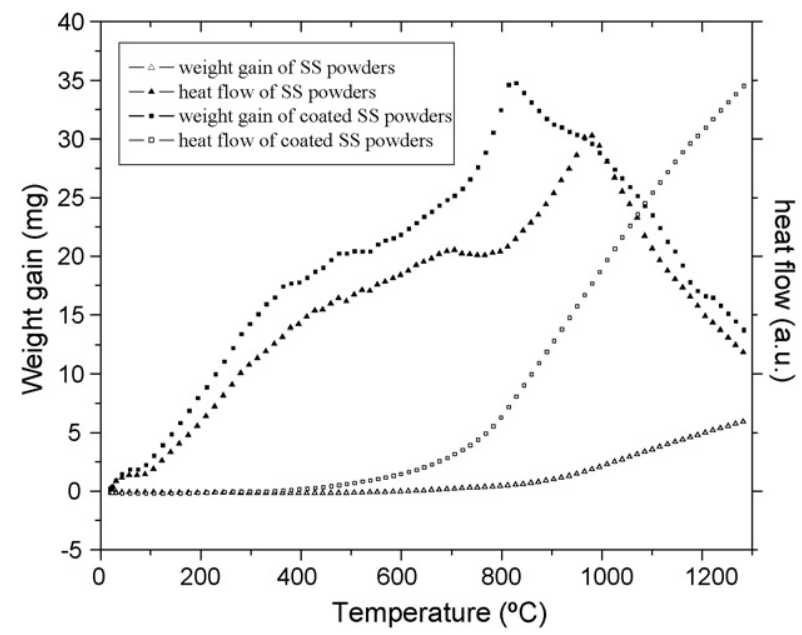

Fig. 3 - Thermal analysis (DTA-TG) of the coated and uncoated powders.

distinguished by hills and valleys, greatly increasing the area exposed to the surrounding atmosphere; the coatings have intercolumnar channels; the thin films have a nanocrystalline structure.

With regard to the ability of coated powders to decrease the viscosity of the feedstocks for injection moulding of stainless steel powders, the critical powder volume concentration values were estimated by torque rheometry by monitoring the

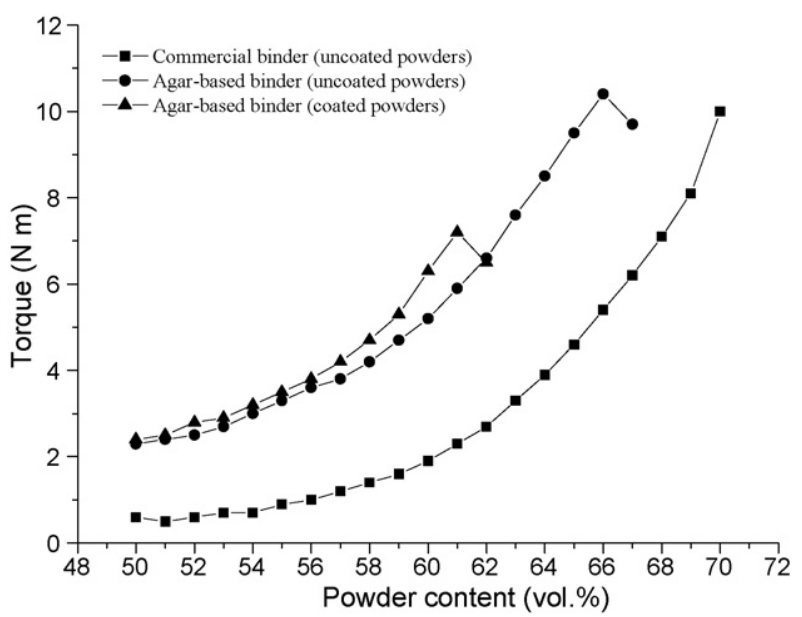

Fig. 4 - Mixing torque versus powder content for coated and uncoated powders and two different binders.

torque variation during their mixing with the agar-based and the commercial binders (Liu et al., 2005). Tests with powder concentrations varying from 50 to $70 \%$ were carried out at the pre-programmed temperatures defined for each binder studied. The relationship between the mixing torque and the powder content of the different feedstocks is shown in Fig. 4. The CPVC corresponds to the powder content where the torque value becomes very high, which means the viscos- (a)

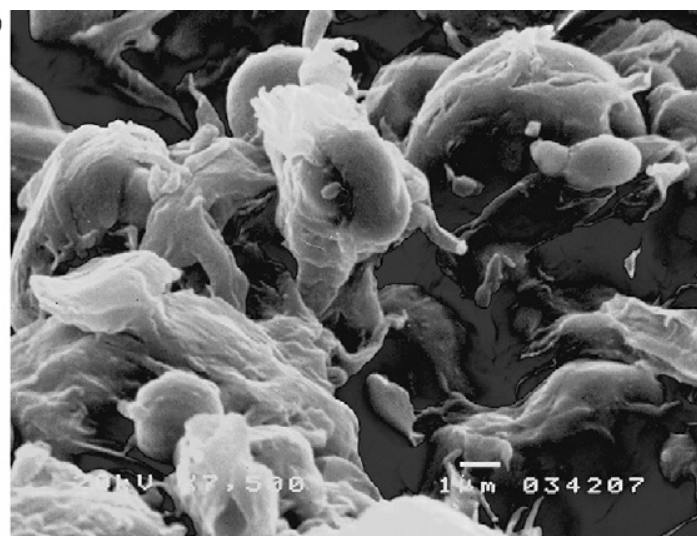

(b)

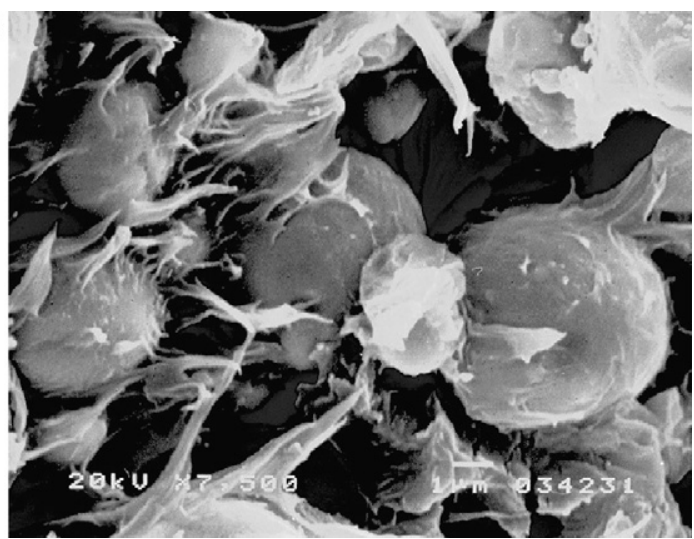

Fig. 5 - Morphology of the commercial binder feedstocks using uncoated (a) and $10 \mathrm{~h}$ coated (b) stainless steel powders.

(a)

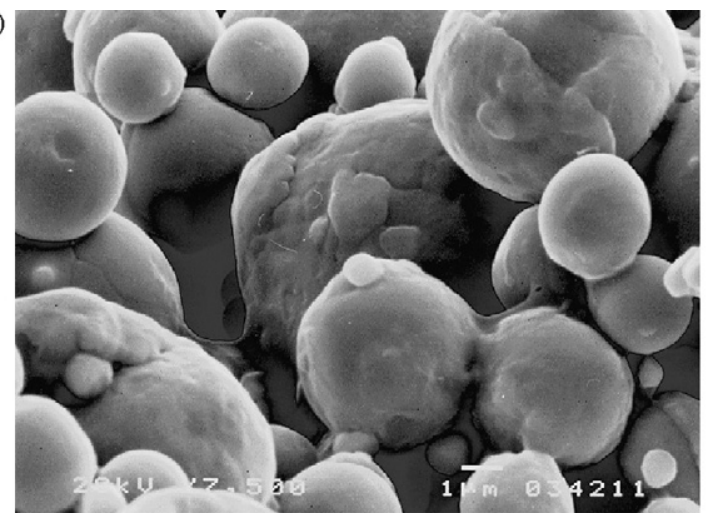

(b)

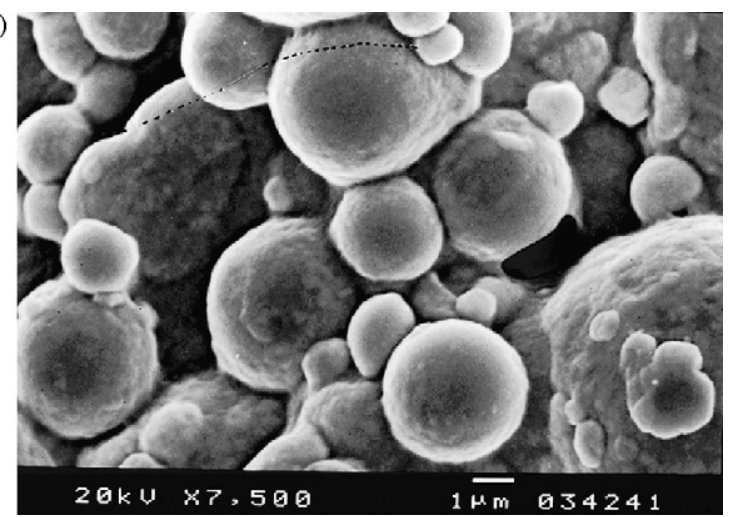

Fig. 6 - Morphology of the agar-based binder feedstocks using uncoated (a) and $8 \mathrm{~h}$ coated (b) stainless steel powders. 
(a)

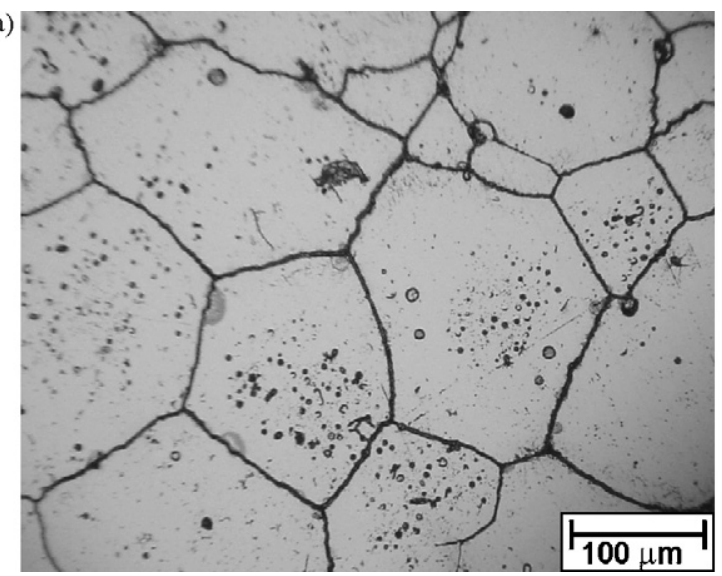

(b)

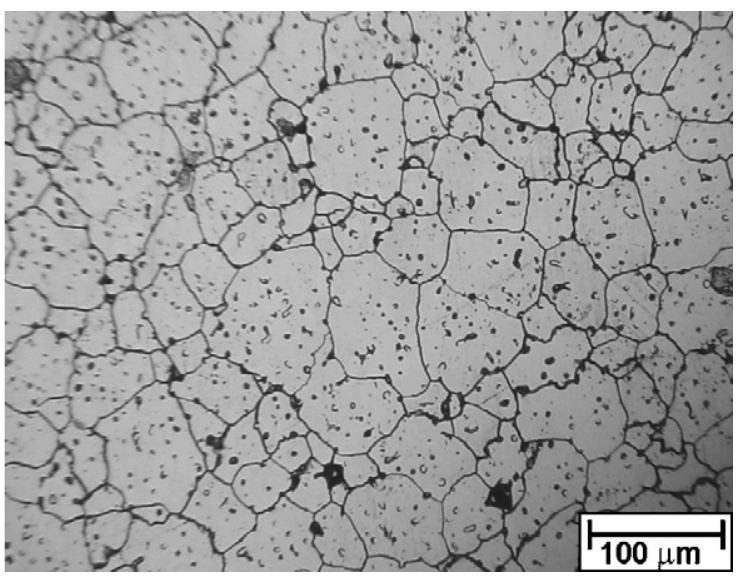

Fig. 7 - Micrographs of sinters from the commercial binder feedstocks with uncoated (a) and $10 \mathrm{~h}$ coated (b) stainless steel powders.

ity is approaching infinity. Thus, the analysis of the results indicates that the most appropriate powder concentration in the mixtures is $57 \%$ for the agar-based binder and $62 \%$ for the commercial one.

According to Fig. 4 no enhancement between coated and uncoated powders has been observed concerning the critical powder volume concentration or the optimal concentration in feedstocks for $\mu$ PIM processing. Nevertheless, the detailed analyses of micrographics of coated and uncoated powders presented in Figs. 5 and 6 reveal a significant improvement in the binder distribution for coated powders, particularly in the case of the commercial binder.

The reactivity of the coated powders, which is essential to decrease the sintering temperature, limits the reaction of chromium with carbon from surrounding atmosphere to the grain boundary (Fig. 7). In fact, the enormous surface/volume ratio resulting from the typical surface morphology associated with the nanocrystallinity of the coating decreases the loss of chromium inside the grains that induces the sensitizing effect, dispersing the precipitation throughout the grain boundaries (Totten, 2007). During the sintering process the presence of coatings on powders has also as main role the control of grain size grows (Fig. 7). The decrease in coating thickness for the lowest values will overcome some difficulty observed in the elimination of pores during sintering of thicker coatings, due to the difficulty of diffusion created by the precipitation in grain boundaries.

\section{Conclusions}

The results demonstrate that the presence of a stainless steel sputtered thin film on the powder's surface results in a homogenous and total coating of powders, particle by particle, and in some cases of agglomerated powders with an "artichoke/blunter" topography and a nanocrystalline surface. Besides the increase in the flowability of powders due to the decrease in interparticulate friction dur- ing the handling of coated powders, the modification of their surface by a deposited coating changes other surface characteristics important for improving a powder's quality with respect to industrial $\mathrm{P} / \mathrm{M}$ processes. Such coated powders could have an important role in the final quality of austenitic stainless steel processed in PIM and $\mu \mathrm{PIM}$.

\section{Acknowledgement}

The authors gratefully acknowledge the Portuguese Foundation for Science and Technology (FCT) under the contract POCTI/CTM/59905/2004 for the financial support provided to undertake this work.

\section{REFERENCES}

Castanho, J.M., Vieira, M.T., Matos, M., Trindade, B., 2007. Nanostructured coated powders for structural net shape components. J. Alloys Compd. 434-435, 383-385.

Fernandes, C.M., Ferreira, V.M., Senos, A.M.R., Vieira, M.T., 2003. Stainless steel coatings sputter-deposited on tungsten carbide powder particles. Surf. Coat. Technol. 176, 103-108.

Fu, G., Loh, N.H., Tay, B.Y., Tor, S.B., Murakoshi, Y., 2004. Replication of metal microstructures by micro powder injection molding. Mater. Des. 25, 729-733.

German, R.M., Bose, A., 1997. Injection Molding of Metals and Ceramics. Metal Powder Industries Federation.

Gioia, A., 1980. Flodex Technical Bulletin. Hanson Research Corporation.

Hartwig, T., Veltl, G., Petzoldt, F., Kunze, H., Scholl, R., Kieback, B., 1998. Powders for metal injection molding. J. Eur. Ceram. Soc. $18,1211-1216$.

Liu, Z.Y., Loh, N.H., Tor, S.B., Khor, K.A., Murakoshi, Y., Maeda, R., Shimizu, T., 2002. Micro-powder injection molding. J. Mater. Process. Technol. 127, 165-168.

Liu, L., Loh, N.H., Tay, B.Y., Tor, S.B., Murakoshi, Y., Maeda, R., 2005. Mixing and characterisation of 316L stainless steel feedstock for micropowder injection molding. Mater. Character. 54, 230-238. 
Liu, L., Loh, N.H., Tay, B.Y., Tor, S.B., Murakosh, Y., Maeda, R., 2007. Effects of thermal debinding on surface roughness in micro powder injection molding. Mater. Lett. 61, 809-812.

Pujari, V.K., 1989. Effect of powder characteristics on Compounding and Green Microscopy in injection moulding process. J. Am. Ceram. Soc. 72, 1981-1984.
Scarlett, B., 2002. Particle Technology the best is yet to come "Haffacheidsrede" 6 Mars, 2002, TU Delft, Faculty of Applied Science, Netherlands.

Totten, G.E., 2007. Steel, Heat Treatment. Taylor\&Francis. Zauner, R., 2006. Micro powder injection moulding. Microelectron. Eng. 83, 1442-1444. 